\title{
IFIP WG 13.2 Workshop on User Experience and User-Centered Development Processes
}

\author{
Marco Winckler ${ }^{1}$, Regina Bernhaupt ${ }^{1}$, Peter Forbrig ${ }^{2}$ and Stefan Sauer ${ }^{3}$ \\ ${ }^{1}$ ICS-IRIT, Université Toulouse 3, 118 route de Narbonne, F-31062 Toulouse, France \\ \{winckler, bernhaupt\} @irit.fr \\ ${ }^{2}$ Universität Rostock, Albert-Einstein-Straße 22, Raum 266, Rostock, Germany \\ peter.forbrig@uni-rostock. de \\ ${ }^{3}$ Universität Paderborn, s-lab, Zukunftsmeile 1, 33102 Paderborn, Germany \\ saueres-lab.uni-paderborn. de
}

\begin{abstract}
This workshop focusses on the interplay of user experience (UX) and user-centered development processes of interactive systems. It is organized by the IFIP Working Group 13.2 on Human-Centered Software Methodologies. It is proposed as a follow-up activity started at an interactive session organized at HCSE 2014. Our ultimate goal is bringing together researchers and practitioners to discuss real-life case studies featuring success and/or failure stories of development processes that take into account UX as an important dimension for the interactive system at concern. Based on these discussions, we expect to deepen the understanding of problems and challenges when dealing with UX in the software development process.
\end{abstract}

\section{Overview and goals}

This workshop is motivated by discussions held during an interactive session featuring an open panel organized during the $5^{\text {th }}$ International Conference on HumanCentered Software Engineering (HCSE 2014) which took place in Paderborn, Germany from September 16-18, 2014 [1], where conference participants were invited to formulate questions/comments about problems they are actually experiencing with the development of interactive systems. Questions and comments provided by participants have revealed a two-fold concern involving UX in development processes for the development of interactive systems and, in particular, agile methods. On one hand, it is widely agreed that UX dimensions are subjective, dynamic and contextdependent [2][3]. For that, existing methods for investigating the impact of UX on the system acceptance often require direct participation of end-users who can report about the use of the system (or at least an advanced prototype) at investigation. On the other hand, agile methods [4] have become popular among software engineers and start to influence the development process of interactive software in industry, in particular by accelerating the cadence of iterations along the development lifecycle and promoting communication mechanisms among members of the development team to reinforce transparence in the decision chain. Nonetheless, UX and agile practices are not always straightforward and pose tricky questions such as: 
- How do we have to change software engineering processes, including agile, to support effectively UX?

- How can UX activities, which are dependent on user studies, be synchronized with software development activities based on fast sprints?

- Should a role UX expert be added in agile processes? Which roles are needed?

- Which process should one follow when UX is in conflict with other user interface properties (such as usability, dependability, privacy...)?

- How can we convince organizations to adopt user-centered design (UCD) activities and incorporate them in organizational processes?

The ultimate goal of this workshop is to deepen the understanding of the current practice of development of interactive software and identify opportunities for improving development processes.

\section{Target audience and expected outcomes}

This workshop is open to everyone (researchers and practitioners) who are interested in UCD processes, and in particular those who have interests in UX and agile methods. As for practitioners, we invite all possible participants in the development process who may include developers, designers, human factor analysts, stakeholders, etc. As for researchers, we expect a high participation of the members of IFIP WG 13.2 and attendees of HCSE 2014 who contribute to the topics of this workshop. We invite participants to present position papers describing real-life case studies featuring success and/or failure stories, and their experiences using methods and tools for dealing with UX along the development. Experiences with any kind of interactive systems are welcome. Contributions on agile methods are welcome, but we also expect to discuss experiences with any type of development process in use. Position papers will be published in official adjunct conference proceedings at the University of Bamberg Press. In the long run, we also expect to compile individual contributions to feature a book such as it has been done in the past [5] for consolidating the knowledge about UX methods and software development.

\section{References}

1. Sauer, S., Bogdan, C., Forbrig, P., Bernhaupt, R., Winckler, M. (Eds.): Human-Centered Software Engineering $-5^{\text {th }}$ IFIP WG 13.2 International Conference, HCSE 2014, Paderborn, Germany, September 16-18, 2014. Springer LNCS 8742, 2014.

2. Law, E., Van Schaik, P.: Modelling user experience - An agenda for research and practice. Interacting with Computers, 22(5), 2010, pp. 313-322.

3. Karapanos, E., Zimmerman, J., Forlizzi, J., Martens, J.-B.: Measuring the dynamics of remembered experience over time. Interacting with Computers, 22(5), 2010, pp. 328-335.

4. Cohn, M.: User Stories Applied: For Agile Software Development. Addison-Wesley Professional, 1st. edition, 304 p., 2004.

5. Seffah, A., Gulliksen, J., Desmarais, M. C. (Eds.): Human-Centered Software Engineering - Integrating Usability in the Software Development Lifecycle. Springer Netherlands, 2005, pp. 17-36. 